\title{
NOVEL CONTROL APPROACH FOR OPTIMAL POWER FLOW IN HYBRID WIND-PHOTOVOLTAIC-DIESEL GENERATION SYSTEMS
}

\author{
DIEP THANH THANG ${ }^{1}$, NGUYEN PHUNG QUANG ${ }^{1}$, NGUYEN DUC HUY $^{2}$ \\ ${ }^{1}$ Institute for Control Engineering and Automation; thang.diept@gmail.com \\ ${ }^{2}$ School of Electrical Engineering, Ha Noi University of Science and Technology
}

Crossref Similarity Check

\begin{abstract}
The paper deals with a formulation of an analytical control model for optimal power flow in an islanded microgrid (MG). In an MG with load change, wind power fluctuation, sun irradiation power disturbance, that can significant influence the power flow, and hence the power flow control problem in real life system faces some new challenges. In order to maintain the balance of power flow, a diesel engine generator (DEG) needs to be scheduled. The objective of the control problem is to find the DEG output power by minimizing the total cost of energy. Using the Bellman principle, the optimality conditions obtained satisfy the Hamilton-Jacobi-Bellman equation, which depends on time and system states, and ultimately, leads to a feedback control or to the so called energy management to be implemented in a SCADA system.
\end{abstract}

Keywords. Microgrid, photovoltaic, wind power, diesel power, optimal power flow, HamiltonJacobi-Bellman equation, distributed generation.

\section{INTRODUCTION}

Due to climate change, renewable electricity such as wind and solar actually plays an important role in providing electrical energy to islands such as microgrid, distributed generations (DGs) have been installed using small-scale power generation technologies and rapidly increased in many countries at lower cost and higher efficiency. However, the uncontrollable nature of wind, solar power as well as load change raises uncertainty for power system operation on one hand, the integration of DGs and the information and communication technology (ICT) into the system is still complex on the other hand. To deal with these issues, the examination of impact of distributed generation on the power fluctuations from penetration of wind, photovoltaic power is presented in $[14,15,18]$. In the works $[4,6,17]$, the authors have considered the hybrid power system whose the energy storage/thermal unit has a high potential for providing regulation power to meet the reverse requirements. Recently, one of important works presented in [16], which examines the optimal problem as the investigation of optimal power flow by adopting the interval algebra and optimization in which the wind power is defined in range of values. Thus the DC power flow problem can be formulated as a non-convex and nonlinear programming. In consequence, to ensure system reliability, the forecasting uncertainty must be considered in short time, for instance twenty-four hours. More importantly, the contribution in [16] is a landmark for class of optimal power flow problem. Concerning the load flow problem which is typically formulated as a set of non-linear 
equations based on constraints of bus voltages has taken some advantage. Such stochastic demand has been developed and presented in [9, 19, 21], those authors used stochastic, fuzzy, and probability programming techniques to model the uncertainties. Although the works in $[9,19,21]$ have been specified to load flow problem with some algorithms, the aforementioned methods are typically dependent on probability model. Furthermore, probability model is really not the real world because it is built from data availability and stochastic nature of the uncertainty in the past and it can not predict accurate probability in the future. The research in all these directions was based on non-convex, and non-linear programming and the DG units are always available. Moreover, in the industrial systems the power system is the most complex for installation, integration, and operation. In fact, the operation of these DG units have different scenarios, that is not because of the strategies of the electricity producer but because of the needs of the customers. Therefore, there are some uncertainties to solve as stochastic modeling of system that becomes of great interest. In order to deal with uncertainties, their sources must be considered at small-scale time such as a certain hour of the day that may affect the modeling and evaluation of the system capacity.

In this paper, we investigate the power flow under uncertainties by minimizing the cost of electricity production. Therefore utilizes the energy balance equation in real time to formulate the optimal power flow problem as optimal control problem of a linear system by using dynamic programming. To do this, it will be expedient to derive an algorithm similar to the Bellman principle where the optimality conditions satisfy Hamilton-Jacobi-Bellman equations, and the value function is convex. In addition, one of the principle reasons for introducing feedback into an optimal control for power flow problem is to make the resulting system relatively insensitive to fluctuations that can deal with uncertainties of power system considered.

\section{STUDY OBJECT}

In this study, we consider the hybrid power system on the island $\mathrm{AC}$ microgrid including AC loads, photovoltaic (PV), wind turbine generator (WTG), and diesel engine generator (DEG). Figure 1 shows the configuration of an island MG.

As in Figure 1, the system consists of three DG units, that is DG1 (as synchronous generator), DG2 (as asynchronous machine), and DG3 (PV source). The DGs are connected to AC bus by power electronic devices used synchronization as AC sources as DEG, WTG, and PV with invert DC voltages into AC called inverter. In addition, PV and WTG are stochastic sources, for they are either locally dispatchable or non-dipatchable and make use of non-controllable primary energy source. On the other hand, the DEG is used for the conversion of mechanical energy into electrical energy as dispatchability [12]. We summarize the characteristic of considered MG in the Table 1.

In what follows, we describe the modeling of the considered microgrid. First of all, the total power generation of DGs satisfies the demand

$$
P_{D E G}(t)+P_{W T G}(t)+P_{P V}(t)=D(t),
$$

where $D(t)$ refers to the load demand at time $t ; P_{W T G}(t)$ is the non-dispatchable (WTG) 


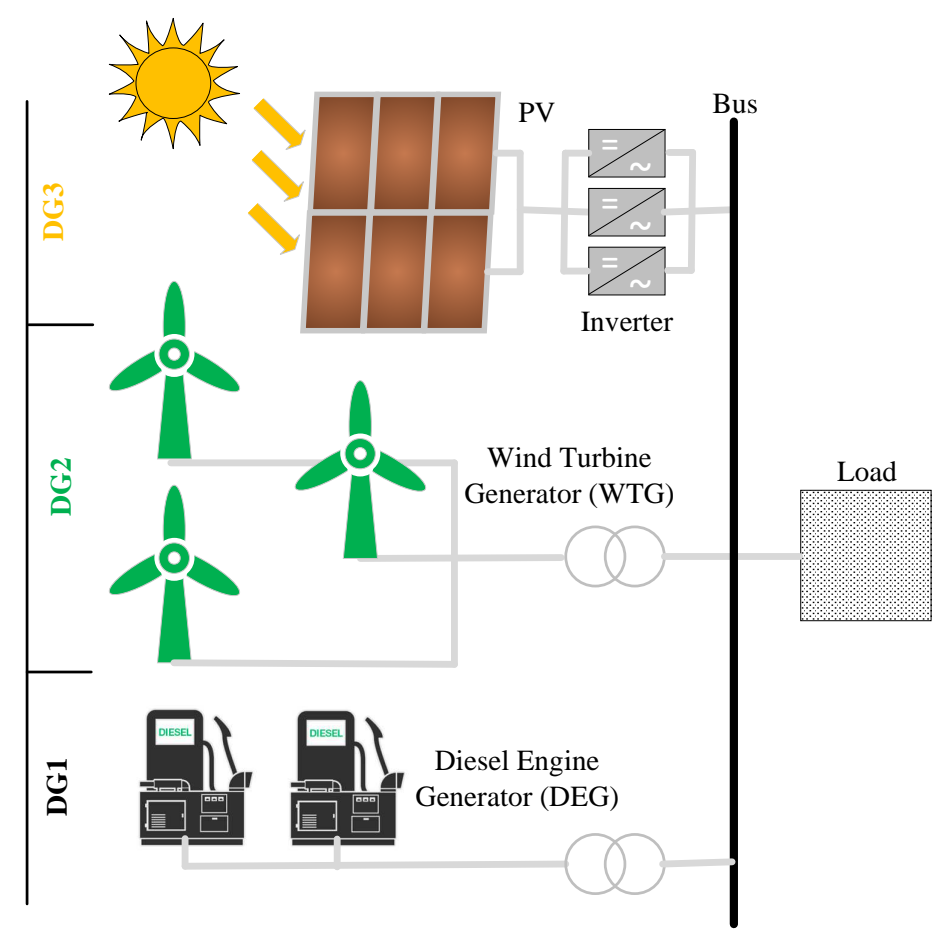

Figure 1. Simplified schematic of an islanded microgrid

Table 1. Classification of MG

\begin{tabular}{|c|c|c|c|}
\hline No & Description & Dispatchability & Stochastic \\
\hline 1 & Diesel engine generator & $\bullet$ & \\
\hline 2 & Wind turbine generator & & $\bullet$ \\
\hline 3 & Photovoltaic & & $\bullet$ \\
\hline 4 & Load & & $\bullet$ \\
\hline
\end{tabular}

output power generation at time $t ; P_{P V}(t)$ is the non-dispatchable $\mathrm{PV}$ output power generation at time $t$; and $P_{D E G}(t)$ is the dispatchable DEG output power generation at time $t$. As mentioned in Table 1, the produced power by PV and WTG depends on the environmental conditions, and the demand depends on the power consumption habits. Hence, the fluctuations in loads, PV, and WTG output power are adjusted by control in the DEG output power. Therefore, the expression of the power balance in equation (1) for randomness in such DG associated with demand can be described as follows

$$
P_{D E G}(t)=D(t)-\left(P_{W T G}(t)+P_{P V}(t)\right) .
$$

The power generation of DEG must evolve the fluctuations in loads and DGs in equation (2). In literature the optimal power flow (OPF) problem has been investigated based on linear and non-linear programming such as $[1,4,5,6,11,16]$. In contrary, we will formulate the considered problem as an optimal control one in order to find out the optimal policies for operation of microgrid in the next section. 


\section{MATHEMATICAL FORMULATION}

In this section, we consider an optimal control problem for the system described in Section 2, and assume that the DEG is always available in continuous time. The problem is considered in finite time (i.e., $0<T<\infty$ ) in order to find the DEG output power. Clearly, to formulate a new model, we shall assume that the power generation in the real time, and let $X(t)$ be the difference between cumulative production and cumulative demand, called surplus at time $t$ if $X(t)$ is positive and backlog if $X(t)$ is negative. They satisfy the one-dimentional differential equation

$$
\begin{gathered}
\frac{d X(t)}{d(t)}=U(t)-\left(D(t)-\left(P_{W T G}(t)+P_{P V}(t)\right)\right), \\
\frac{d X(t)}{d(t)}=f(t, X(t), U(t))
\end{gathered}
$$

and the constraint

$$
\begin{gathered}
P_{\min }^{W T G}(t) \leq P_{W T G}(t) \leq P_{\max }^{W T G}(t), \\
P_{\min }^{P V}(t) \leq P_{P V}(t) \leq P_{\max }^{P V}(t), \\
P_{\min }^{D E G}(t) \leq P_{D E G}(t) \leq P_{\max }^{D E G}(t),
\end{gathered}
$$

where $U(t)=P_{D E G}(t)$ is the control variable in equation (2) and $U(t) \in \mathbb{R}^{+}=[0,+\infty)$ in $[k W], P_{W T G}(t), P_{P V}(t)$ and $P_{D E G}(t)$ are within their forecasted upper bounds $P_{\max }^{W T G}(t)$, $P_{\max }^{P V}(t), P_{\max }^{D E G}(t)$ and lower bounds $P_{\min }^{W T G}(t), P_{\min }^{P V}(t), P_{\min }^{D E G}(t)$ (in $\left.[k W]\right), X(t)$ is energy in $[k W h]$ and $X(t) \in \mathbb{R}=(-\infty,+\infty), f(t, X, U)$ is the state function and satisfies the Lipschitz condition

$$
\left|f\left(t, X_{1}, U\right)-f\left(t, X_{2}, U\right)\right| \leq K_{p}\left|X_{1}-X_{2}\right|
$$

where $K_{p}$ is constant.

The behavior of the state variable $X(t)$ will be specified shortly in the subsection 3.2.

Let us define cost function (cost-to-go or cost-to-arrive) by

$$
J(t, X ; U)=\int_{t}^{T} G(s, X(s), U(s)) d s,
$$

where $G(t, X(t), U(t))$ is the running cost function: $G()=.C^{+} X^{+}+C^{-} X^{-}$with $C^{+}$representing a unit surplus cost at time $t, C^{-}$the unit backlog cost at time $t, X^{+}=\max (X, 0)$, and $X^{-}=\min (0,-X)$.

Thus, the function $J(t, X ; U)$ is called an overall cost of the system. To simplify things, we make the following assumptions in this paper to describe the hybrid power system:

(A.1) The total power generation satisfies the power demand in finite time considered $T$, i.e, at any time $t$ 


$$
P_{D E G}(t)+P_{W T G}(t)+P_{P V}(t) \geq D(t) .
$$

(A.2) The stochastic power values $D(t), P_{W T G}(t)$ and $P_{P V}(t)$ are forecasted values in $[k W]$.

\section{Definition 3.1.}

(1) A control variable $Y(t, X)=\{U(t, X)=U(t) \geq 0\}$ is called an admissible control;

(2) A control $\Omega(t, X)$ is the set of admissible control $Y$ with initial vector $X(t)=X$.

Our motivation is to obtain admissible control $U(t, X) \in \Omega(t, X)$ that optimizes the cost function (9). In what follows, we will build the model that satisfies the contrary (3-8) and optimization of (9) by using the dynamic programming approach.

We formulate the power flow problem defined above. Under appropriate conditions, the optimal control policy is to satisfy (3-8) in order to determine the OPF $U(t, X)$ which minimizes the cost function described in (9). These policies are characterized by a target production level subject to capacity constraints.

We denote by $v(t, X)$ the value function, i.e.

$$
v(t, X)=\inf _{U(.) \in \Omega(t, .)} J(t, X ; U)
$$

This function will be used to establish the optimality conditions. For simplicity in the presentation of the model, we use only the sign $v(t, X)$. Based on the dynamic programming principle, the following theorem is used for the generalization of the value function in (10).

Theorem 3.1. Control problem satisfies the system of partial differential equations

$v(t, X)=\inf _{U(.) \in \Omega(t, .)}\left\{G(t, X, U)+v_{t}(t, X)+\left(U(t, X)-\left(D(t)-P_{W T G}(t)-P_{P V}(t)\right)\right) v_{x}(t, X)\right\}$

at time $t$ the initial and boundary conditions are satisfied

$$
\begin{gathered}
X(t)=X \text { for }(t, X) \in Q, \\
\\
v(T, X(T))=0,
\end{gathered}
$$

where the terms $v_{t}(t, X)$ and $v_{X}(t, X)$ denote the gradient of value function with respect to time $t$ and state variable $X$, respectively, and $Q=\left[t_{0}, T\right] \times \mathbb{R}$.

Proof. The proof of this theorem is developed in [13] as well as in [2].

The following theorem presents the necessary and sufficient conditions for which an optimal solution exists.

Theorem 3.2. Let $v(t, X) \in Q$ be a solution to (8). Then for all $(t, X) \in Q$

(i) For every admissible control system $U(t, X)$

$$
v(t, X) \leq J(t, X ; U)
$$


(ii) If there exists an admissible system $U^{*}(t, X)$ such that

$$
U^{*}(t, X) \in \underset{U(.) \in \Omega(.)}{\operatorname{argmin}}\left\{G(t, X, U)+f(t, X, U) v_{X}(t, X)\right\}
$$

almost everywhere in $t$, then $v(t, X)=J\left(t, X ; U^{*}\right)$, and $U^{*}(t, X)$ is the optimal solution.

Proof. The Proof of this theorem is developed from the results in page 8 of [7].

Remarks. The system of partial differential equations (11) is known as the Hamilton-JacobiBellman (HJB) equations associated with optimal control problem under study. The optimal feedback control (11) is designed to drive the system to the optimal point (hedging point or balance point), and corresponds to the value function described by equation (11). Then, when the value function $v(t, X)$ is available, an optimal policy can be obtained as in equation (14). However, an analytical solution of equation (11) is so hard to find. Thus, the numerical solution of HJB equation (14) adopted from Kushner's method [10] is represented in the section 4 .

\subsection{The feedback control}

Let $w(t)$ be the random parameter such as the stochastic wind power, demand, or solar radiation (called disturbance or noise dependent on context). Let $\mu(t, X(t))$ be the mapping of $U(t, X)$ such that $\mu(t, X(t)) \in \omega(t, X)$. In the equation (3), the disturbance $w(t)$ may consist of $D(t), P_{W T G}(t)$, and $P_{P V}(t)$. Hence, the feedback control of (3) is represented in the Figure 2.

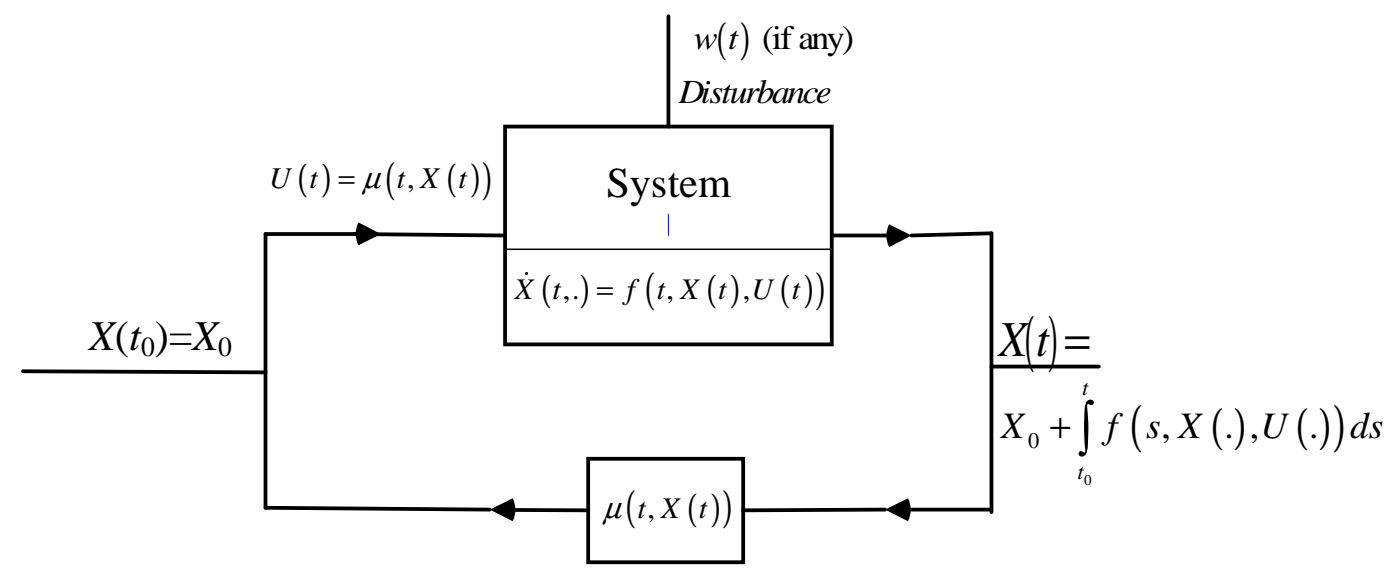

Figure 2. The closed-loop policies [2] 


\subsection{Behavior of cumulative production}

By definition, the energy in equation (3) whose value is described as follows

$$
X(t)=X_{0}+\int_{t_{0}}^{t}\left[U(t, X)-\left(D(s)-\left(P_{W T G}(s)+P_{P V}(s)\right)\right)\right] d s .
$$

In the Figure 3, there are three regions: (1) the total power generation is more than the demand in the interval $\left[t_{1}, t_{2}\right],(2)$ it is less than the demand when $t<t^{*}$, and (3) it is probably in equality in the interval $\left(t^{*}, t_{1}\right)$. The balance point (hedging point) is at time $t^{*}$ where the production meets the demand.

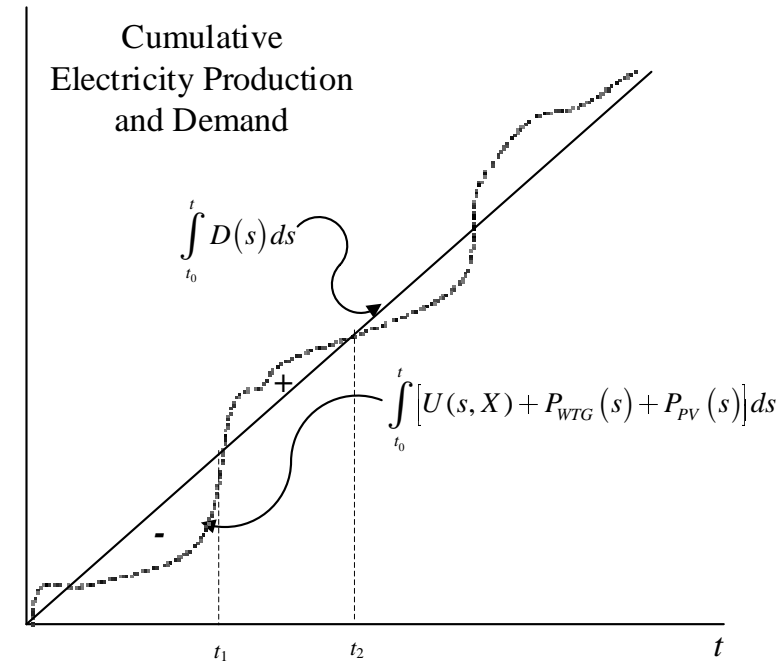

Figure 3. Energy production strategy*

(*this behavior is modified from Gershwin's framework in [8] for the case of production control with stochastic events.)

\section{NUMERICAL APPROACH}

In this section, we develop the numerical method for solving the optimality conditions represented in the previous section. This method is based on Kushner's approach [10]. By adopting the algorithm in [3], the HJB equation (11) which includes the gradient of value function of $v(t, X)$ can be solved. Let $\Delta X>0$ and $\Delta t>0$ denote the length of the finite difference interval of the variable $X$ and $t$ respectively. The first-order partial derivatives of the value functions $v_{t}($.$) and v_{x}($.$) in equation (11) are replaced by the following expressions$

$$
\begin{gathered}
v_{t}(t, X)=\frac{v(t+\Delta t, X)-v(t, X)}{\Delta t}, \\
v_{t}(t, X)= \begin{cases}\frac{v(t, X+\Delta X)-v(t, X)}{\Delta X} & \text { if } f(t, X, U) \geq 0, \\
\frac{v(t, X)-v(t, X-\Delta X)}{\Delta X} & \text { otherwise. }\end{cases}
\end{gathered}
$$


Using $\Delta X$ and $\Delta t$, and after manipulations, the HJB equations can be rewritten as follows

$$
\begin{aligned}
v^{\Delta}(t, X)=\min _{U^{\Delta}(.)}[G(.) & +\frac{v^{\Delta}(t+\Delta t, X)-v^{\Delta}(t, X)}{\Delta t} \\
& \left.+\left(U^{\Delta}-\left(D-\left(P_{W T G}+P_{P V}\right)\right)\right) \frac{( \pm) v^{\Delta}(., X \pm \Delta X) \mp v^{\Delta}(.)}{\Delta X}\right] .
\end{aligned}
$$

The next theorem shows that $v^{\Delta}(t, X)$ is an approximation to $v(t, X)$ for small step size $\Delta X$.

Theorem 4.1. Let $v^{\Delta}(t, X)$ denote a solution to HJB equation (18). Assume that there are constants $C_{g}$ and $K_{g}$ such that

$$
0 \leq v^{\Delta}(t, X) \leq C_{g}\left(1+|X|^{K_{g}}\right)
$$

then

$$
\lim _{\Delta \rightarrow 0} v^{\Delta}(t, X)=v(t, X)
$$

Proof. The proof of this theorem is adopted from the one in [20] for the case of deterministic control problem.

In this study, we make use of the policy improvement technique to obtain a solution of the approximating optimization problem. Let $G_{X}^{\Delta}$ and $G_{U}^{\Delta}$ be the grids of the states and control vectors belonging to the control space, the algorithm is represented as follows:

Step 1 (Initialization): Choose $\epsilon \in \mathbb{R}^{+}$. Let $k=1$ and $v_{\Delta}^{k}(t, X)=0,(t, X) \in\left[t_{0}, T\right] \times G_{X}^{\Delta}$ and $U^{k} \in G_{U}^{\Delta}$ (initial policy).

Step 2 : For a given $U^{k} \in G_{U}^{\Delta}$, compute

$$
v_{\Delta}^{k-1}(t, X)=v_{\Delta}^{k}(t, X), \vee(t, X) \in\left[t_{0}, T\right] \times G_{X}^{\Delta} .
$$

Step 3 : Compute the corresponding value function to obtain the control policy $U(t, X(t))$.

Step 4 : Convergence test

$$
\begin{gathered}
\delta_{\min }=\min \left|v_{\Delta}^{k}(t, X(t))-v_{\Delta}^{k-1}(t, X(t))\right|, \\
\delta_{\max }=\max \left|v_{\Delta}^{k}(t, X(t))-v_{\Delta}^{k-1}(t, X(t))\right| .
\end{gathered}
$$

If $\left|\delta_{\max }-\delta_{\min } \leq \epsilon\right|$, then stop, else $k=k+1$ and go to the Step 2 .

\section{NUMERICAL EXAMPLE}

The proposed model in Section 3 is for application of hybrid wind / photovoltaic / diesel engine generator generation system such as follows: 


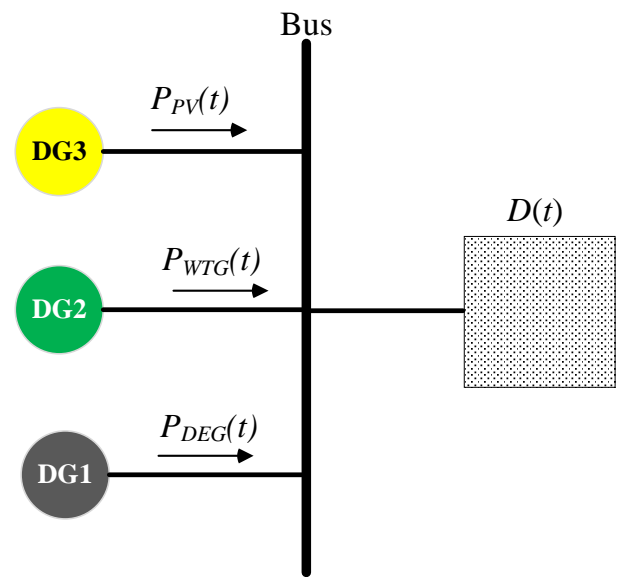

Figure 4. Energy production strategy*

Table 2. Parameters of optimal power flow model

\begin{tabular}{|c|l|c|c|}
\hline No & Description & Lower & Upper \\
\hline 1 & Time interval $[\mathrm{h}]$ & 0 & 24 \\
\hline 2 & WTG power $[\mathrm{kW}]$ & 0 & 500 \\
\hline 3 & PV power $[\mathrm{kW}]$ & 0 & 600 \\
\hline 4 & DEG power $[\mathrm{kW}]$ & 1000 & 3500 \\
\hline 5 & Load demand $[\mathrm{kW}]$ & 0 & 3500 \\
\hline 6 & $C^{-}$Backlog cost $[\$ / \mathrm{kWh}]$ & 10 & - \\
\hline 7 & $C^{+}$Surplus cost $[\$ / \mathrm{kWh}]$ & 1 & - \\
\hline
\end{tabular}

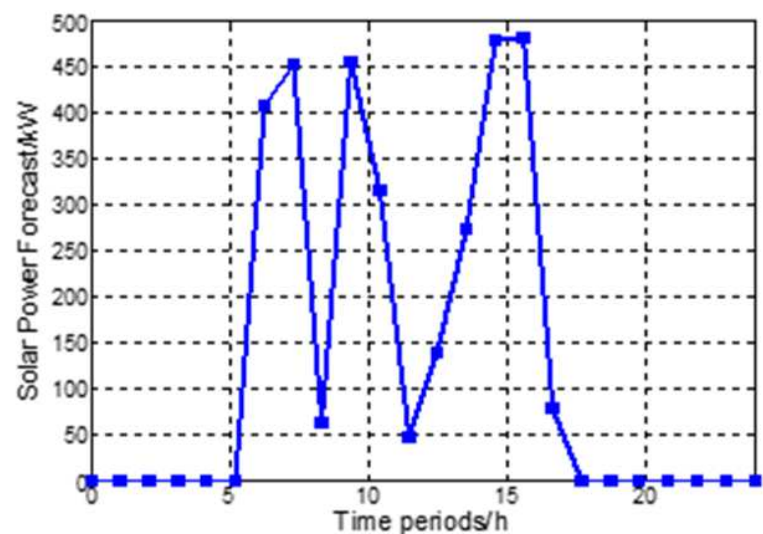

Figure 5. The power flow of MG

Figure 4 presents the power flow of microgrid including DG1 as DEG, DG2 as WTG, and DG3 as PV.

In this example, the forecast of load demand, PV power, and wind power generations are 


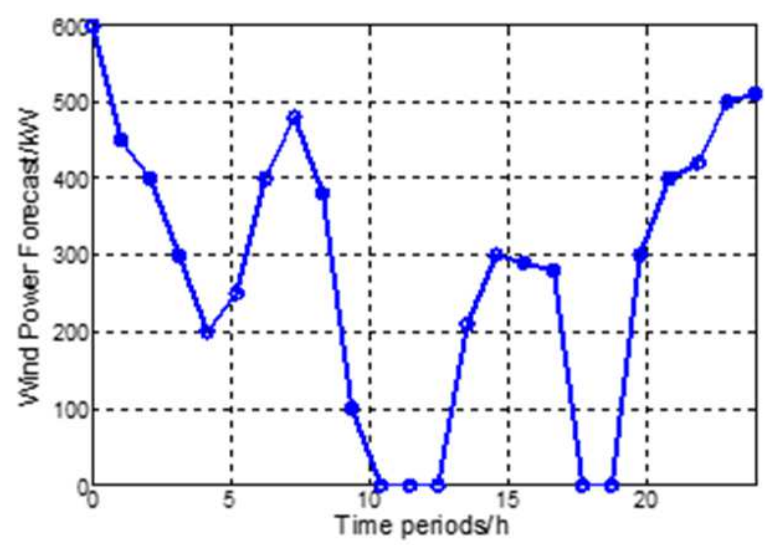

Figure 6. Wind power forecast $[\mathrm{kW}]$

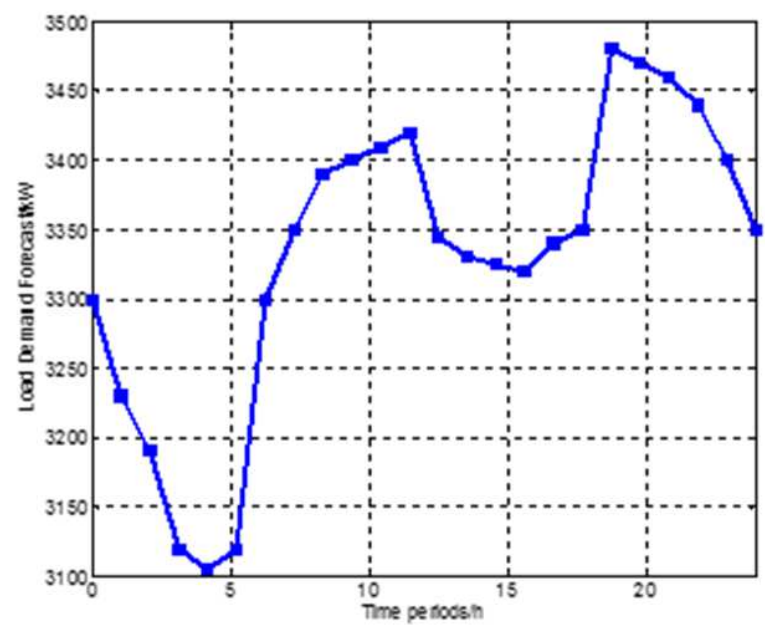

Figure 7. Demand forecast $[\mathrm{kW}]$

represented in Figures 5-7. The constraints of system parameters in equations (4-7) for MG are presented in the Table 2, these parameters also obey the Assumptions A.1 and A.2.

The results are illustrated in Figures 8-9. Figure 8 represents the optimal production of DEG output power versus time $t$ in the interval $[0,24 \mathrm{~h}]$. Figure 9 represents the cumulative electricity production of MG and the load demand versus time $t$. This figure shows that, the effectiveness of optimal control gives the birth to optimal power flow of MG that satisfies the load demand, thus the characteristic is linear instead of being non-linear as in Figure 3.

\section{CONCLUSIONS}

The problem which has been considered in this paper has main objective: optimal power flow control with uncertain power injection. We have formulated a new model as a stochastic control problem by adopting Bellman framework in order to solve production problem with 


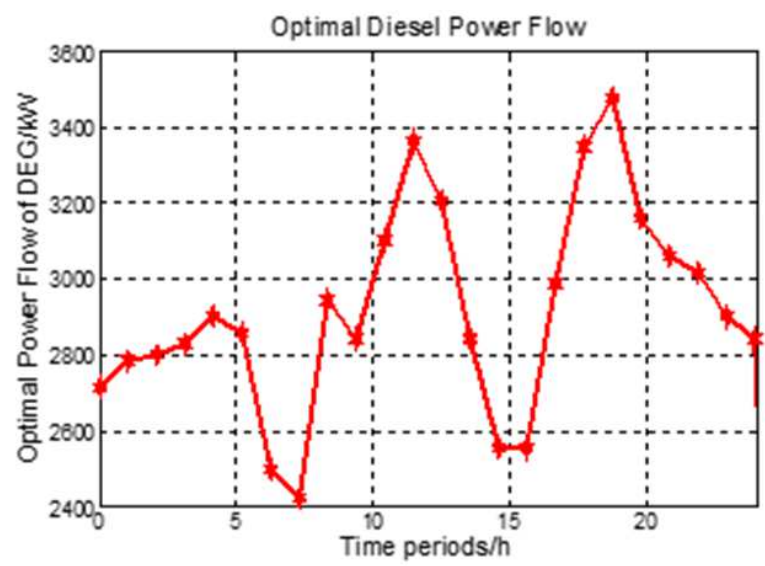

Figure 8. Optimal output power of DEG $[\mathrm{kW}]$

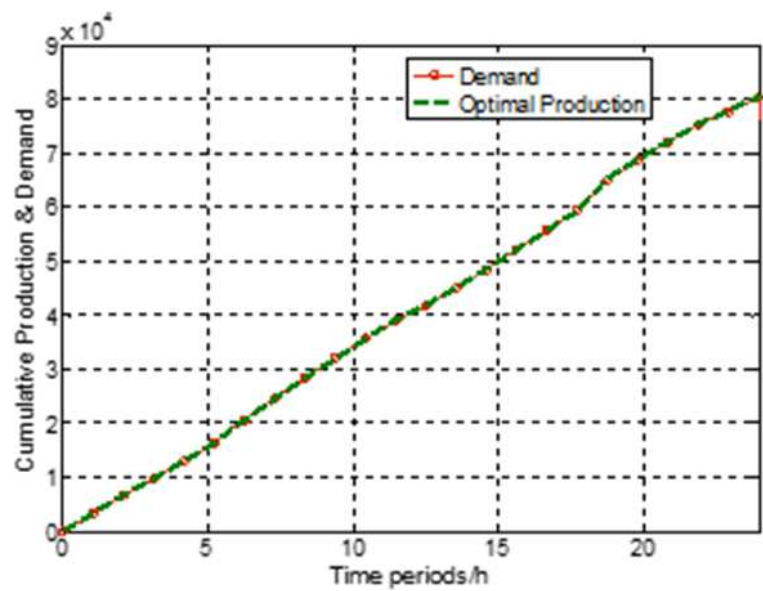

Figure 9. Cumulative production $[\mathrm{kWh}]$

minimizing the surplus cost. The optimality conditions have been established by using dynamic programming as Hamilton-Jacobi-Bellman equation. According to the results, the proposed model makes considered system joint between the optimal power flow and optimal control problem. The new proposed model enables us to solve the supply-demand balancing problem in islanded microgrid that allows interoperability and autonomy on the energy management so called SCADA system. We applied our proposed model to a real life system of an island MG at small-scale with demand, PV power, and WTG power forecast, and the DEG output power is control variable. The results of test system have demonstrated the effectiveness of the proposed method. In the future work, we make use of probability models for unpredictable data such as the stochastic sources (PV and wind power). 


\section{REFERENCES}

[1] F. Alvarado, Y. Hu, and R. Adapa, "Uncertainty in power system modeling and computation," in Proceedings 1992 IEEE International Conference on Systems, Man, and Cybernetics, Oct 1992, pp. 754-760 vol.1.

[2] D. Bertsekas, Ed., Dynamic Programming and Optimal Control, 4th ed. Athena Scientific, Belmont, Massachusetts, 2017, vol. 1.

[3] E. Charlot, J. Kenné, and S. Nadeau, "Optimal production, maintenance and lockout/tagout control policies in manufacturing systems," International Journal of Production Economics, vol. 107, no. 2, pp. 435 - 450, 2007, operations Management in China.

[4] T. Ding, R. Bo, F. Li, Q. Guo, H. Sun, W. Gu, and G. Zhou, "Interval power flow analysis using linear relaxation and optimality-based bounds tightening (obbt) methods," IEEE Transactions on Power Systems, vol. 30, no. 1, pp. 177-188, Jan 2015.

[5] T. Ding, R. Bo, F. Li, and H. Sun, "Optimal power flow with the consideration of flexible transmission line impedance," IEEE Transactions on Power Systems, vol. 31, no. 2, pp. 16551656, March 2016.

[6] W. El-Khattam, Y. G. Hegazy, and M. M. A. Salama, "Stochastic power flow analysis of electrical distributed generation systems," in 2003 IEEE Power Engineering Society General Meeting (IEEE Cat. No.03CH37491), vol. 2, July 2003, p. 1144 Vol. 2.

[7] W. H. Fleming and H. M. Soner, Controlled Markov Processes and Viscosity Solutions, 2nd ed. Springer-Verlag New York, 2006, vol. 25.

[8] S. B. Gershwin, Manufacturing Systems Engineering. Prentice Hall, 1994.

[9] M. K. Kim, D. H. Kim, Y. T. Yoon, S. S. Lee, and J. K. Park, "Determination of available transfer capability using continuation power flow with fuzzy set theory," in 2007 IEEE Power Engineering Society General Meeting, June 2007, pp. 1-7.

[10] H. Kushner and P. G. Dupuis, Numerical Methods for Stochastic Control Problems in Continuous Time, 2nd ed. Springer-Verlag New York, 2001, vol. 24.

[11] J. Lee, J. H. Kim, and S. K. Joo, "Stochastic method for the operation of a power system with wind generators and superconducting magnetic energy storages (smess)," IEEE Transactions on Applied Superconductivity, vol. 21, no. 3, pp. 2144-2148, June 2011.

[12] G. Papaefthymiou, P. Schavemaker, L. van der Sluis, W. Kling, D. Kurowicka, and R. Cooke, "Integration of stochastic generation in power systems," International Journal of Electrical Power \&3 Energy Systems, vol. 28, no. 9, pp. 655 - 667, 2006, selection of Papers from 15th Power Systems Computation Conference, 2005. [Online]. Available: http://www.sciencedirect.com/science/article/pii/S0142061506000846

[13] S. P. Sethi and G. L. Thompson, Optimal Control Theory: Applications to Management Science, 2nd ed. Springer US, 2000.

[14] P. Sorensen, N. A. Cutululis, A. Vigueras-Rodriguez, L. E. Jensen, J. Hjerrild, M. H. Donovan, and H. Madsen, "Power fluctuations from large wind farms," IEEE Transactions on Power Systems, vol. 22, no. 3, pp. 958-965, Aug 2007.

[15] Y. T. Tan and D. S. Kirschen, "Impact on the power system of a large penetration of photovoltaic generation," in 2007 IEEE Power Engineering Society General Meeting, June 2007, pp. 1-8. 
[16] D. Tao, Power System Operation with Large Scale Stochastic Wind Power Integration. Springer Theses, 2017.

[17] K. Trine, "Stochastic programming with applications to power systems," Ph.D. dissertation, Aarhus University, Denmark, 2007.

[18] F. Vallee, J. Lobry, and O. Deblecker, "System reliability assessment method for wind power integration," IEEE Transactions on Power Systems, vol. 23, no. 3, pp. 1288-1297, Aug 2008.

[19] D. Villanueva, J. L. Pazos, and A. Feijoo, "Probabilistic load flow including wind power generation," IEEE Transactions on Power Systems, vol. 26, no. 3, pp. 1659-1667, Aug 2011.

[20] H. Yan and Q. Zhang, "A numerical method in optimal production and setup scheduling of stochastic manufacturing systems," IEEE Transactions on Automatic Control, vol. 42, no. 10, pp. 1452-1455, Oct 1997.

[21] P. Zhang and S. T. Lee, "Probabilistic load flow computation using the method of combined cumulants and gram-charlier expansion," IEEE Transactions on Power Systems, vol. 19, no. 1, pp. $676-682$, Feb 2004.

Received on November 22, 2016

Revised on July 17, 2017 